\title{
Molecular cloning of the mouse CCK gene: expression in different brain regions and during cortical development
}

\author{
M.Vitale, A.Vashishtha', E.Linzer, D.J.Powell ${ }^{2}$ and J.M.Friedman* \\ Howard Hughes Medical Institute and 'Laboratory of Molecular Cell Biology, The Rockefeller \\ University, New York, NY 10021 and ${ }^{2}$ Department of Molecular Embryology, Institute of Animal \\ Physiology and Genetic Research, Cambridge, CB2 4AT, UK
}

Received April 18, 1990; Revised and Accepted July 30, 1990

\begin{abstract}
In this paper we describe experiments that address specific issues concerning the regulation of the mouse cholecystokinin gene in brain and intestine. The mouse cholecystokinin gene was cloned and sequenced. Extensive homology among the mouse, man and rat genes was noted particularly in the three exons and the regions upstream of the RNA start site. RNAse protection assays for each of the three exons were used to demonstrate that CCK is expressed in only a subset of tissues and that the same cap site and splice choices are used in brain, intestine as well as in cerebellum, cortex, midbrain, hypothalamus and hippocampus. CCK RNA was also noted to be detectable in kidney. Thus the same gene using the same promoter is expressed in subsets of cells that differ in their biochemical, morphologic and functional characteristics. The level of expression of CCK was also monitored during mouse cortical development and the appearance of CCK RNA was compared to glutamate decarboxylase (GAD), enkephalin and somatostatin. It was noted that each of these cortical markers was first expressed at different times during cortical development. The appearance of CCK RNA during intestinal development was also measured and found to precede appearance in cortex by several days.
\end{abstract}

\section{INTRODUCTION}

Differentiation viewed at the cellular level is characterized by the production of tissue specific protein and mRNAs in specialized cells (1). In some cases, tissue specific mRNAs are restricted in their distribution to a single cell type. Oftentimes, however, tissue specific mRNAs are present in subsets of tissues (2).

Complex distributions of cell specific markers are particularly evident in the developing nervous system where such markers are frequently present in many different types of neurons at different anatomic loci $(3-5)$. This principle is illustrated by the distribution of the brain-gut peptide cholecystokinin (6).
CCK is a hormone which was first purified from small intestine where its controlled release from endocrine cells in response to intraintestinal lipid was shown to stimulate exocrine pancreatic secretion and gall bladder contraction (7). In 1975 cholecystokinin was found at levels in brain that exceeded those demonstrated in small intestine (8). More recent studies on the regional distribution of CCK in brain have demonstrated that this peptide is present in many different brain regions in subsets of neurons that differ in their biochemical, morphologic and functional characteristics. In cerebral cortex for example CCK is found in a small subset of GABA ergic interneurons $(9,10)$, while in the substantia nigra CCK immunoreactivity has been demonstrated in dopaminergic neurons that project to the limbic system (11). CCK-producing neurons in the hypothalamus have been shown to also produce CRH (12). CCK is also present in many other brain regions although the characteristics of CCK-producing neurons in other loci are not as well described. Furthermore, the other neuronal markers mentioned, GABA, dopamine and $\mathrm{CRH}$, are not restricted in their distribution to CCK-producing cells $(9,11)$. Therefore, CCK-producing neuronal cells in each brain region appear to express a unique combination of genes, including $\mathrm{CCK}$, that by themselves are not restricted to that cell type.

It is our objective to develop a full understanding of the molecular mechanisms by which the CCK gene can be expressed as part of different developmental programs in different cells of the developing brain. Previously we have described the cloning of a mouse CCK CDNA and presented evidence that the same mRNA start sites are likely used in brain and intestine (13). In the present report we describe the molecular cloning of the mouse CCK gene and present definitive evidence that the same RNA start site and splice sites are used in intestine and in different regions of the brain. We also report the expression of cholecystokinin RNA in kidney. Finally, we have compared the developmental expression of the CCK gene in cortex to that of the GABA synthesizing enzyme, GAD, and other cortical neuropeptides, and found that each of several neuron specific markers first appear as mRNA at different times in cortical development.

\footnotetext{
* To whom correspondence should be addressed
} 


\section{MATERIALS AND METHODS}

\section{Cloning of the CCK gene}

$10^{6}$ clones from one of two mouse genomic libraries were plated on $150 \mathrm{~mm}^{2}$ plates and screened using the plaque lift procedure of Benton and Davis. One library was derived from a partial digest with EcoRI cloned into charon $4 \mathrm{~A}$ and the other was derived by cloning MboI partial digests into $\lambda E$ mb13 (14). The library was screened using the plaque lift procedure of Benton and Davis (15). Duplicate nitrocellulose filters were screened with the CCK cDNA and plaque purified. In order to obtain a phage clone that extended further $5^{\prime}$, additional isolates were collected by probing with a $1200 \mathrm{bp} \mathrm{BamHI}$ fragment at a position -6700 to -5500 from the CCK cap site.

In the initial screen, two separate phage clones were isolated four times each. The phage DNAs were restriction mapped and shown to be overlapping. Shotgun subclones of the gene were made using EcoRI, BamHI and BgIII and clones with coding sequence were selected using the colony lift procedures of Grunstein and Hogness with either a mouse cDNA or an oligonucleotide whose sequence 5' CTTAGCTGGACAGC AGCCGTTGG 3' was derived from the published rat CCK first exon $(16,17)$. Clones hybridizing to each of the probes were sequenced using the dideoxy chain termination method (18). Sequences were compared to the mouse cDNA sequence as well as to the rat and human CCK gene using the fastn2 sequence comparison program (19).

\section{RNA preparation and RNAse protection assays}

RNA from tissue or cell lines was prepared using guanidinium thiocyanate technique. Tissues were dissolved in guanidinium thiocyanate and the RNA was pelleted through a cesium chloride cushion (20). RNA was prepared from different brain regions after regional dissection using standard anatomic criteria (21). RNA was also made from dissected cortex at different times of development; pregnancies were timed by the presence of vaginal plugs (= day 1 of pregnancy).

For quantitation of RNA, RNAse protection assays were performed in which equivalent amounts of RNA, as measured by optical density, were hybridized to specific radiolabeled antisense RNA and subsequently trimmed with T2 RNAse. RNAse resistant fragments were resolved on a polyacrylamide gel (22). The hybridization temperature used was $59^{\circ}$ for all probes. To monitor expression of the first exon of CCK, an RNA probe was synthesized from a plasmid containing an Sst-Ssp fragment extending from -800 to +74 which was linearized with Sst and labeled with Sp6 polymerase. Expression of exon 2 of CCK was monitored using a $4.5 \mathrm{~kb}$ EcoRI fragment extending from -2400 to +2100 which was used to generate an antisense RNA with 17 polymerase after digestion with SspI. Expression of the CCK third exon was monitored by generating a 700 bp antisense RNA from a BglII subclone that was linearized with EcoRI and also labeled with $T 7$ polymerase.

Measurement of GAD, somatostatin, enkaphalin and actin was made using antisense probes derived from plasmids kindly provided by Allan Tobin (GAD; 23), Mark Montminy (somatostatin; 24), Steve Sabol (enkephalin; 25) and Tamar Enoch (26). The characteristics of these probes have been previously reported (24-26). In the case of rat enkephalin probe and the rat somatostatin probe, mouse RNA protected the full length rat probe. In the case of GAD (which is feline) and actin (which is human) truncated fragments were protected. The feline GAD probe protected a brain specific RNA of $\sim 310 \mathrm{bp}$ in mouse
(Fig. 6A, unpublished data). The use of the actin probe has been reported previously (27).

\section{Primer extension}

The primer extension assay was performed by hybridizing a 32 bp oligonucleotide of the sequence 5' CAC GCACAG ACATACGCCGCT CTT CATCC CTTT 3 ' to poly A+ RNA from brain and intestine. This is an antisense sequence that begins in the CCK second exon and extends 4 bp into the first exon (see Fig. 2C). The oligonucleotide was radiolabelled with $\gamma^{32}$ ATP and T4 polynucleotide kinase and hybridized to $5 \mu \mathrm{g}$ poly $\mathrm{A}+\mathrm{mRNA}$ at $37^{\circ}$ in $40 \mathrm{nM}$ Pipes pH 6.4, $1 \mathrm{mM}$ EDTA pH 8, $0.4 \mathrm{mM}$ $\mathrm{NaCl}$ and $80 \%$ formamide. This was followed by addition of nucleotides and reverse transcriptase (13). The primer extension products were resolved on a $5 \%$ urea acrylamide gel.

\section{Polymerase chain reactions for CCK cDNA}

One $\mu \mathrm{g}$ of brain RNA prepared as described was reverse transcribed using 200 units MoMuLV reverse transcriptase in $1 \times$ PCR buffer $(50 \mathrm{mM} \mathrm{KCl}, 20 \mathrm{mM}$ Tris-Cl pH 8.4, $1 \mathrm{mM}$ $\mathrm{MgSO}_{4}$ ) and $100 \mathrm{ng}$ of the oligonucleotide complementary to sequences of the $3^{\prime}$ untranslated region, by incubation for $10 \mathrm{~min}$ at room temperature then $30 \mathrm{~min}$ at $92^{\circ} \mathrm{C}$. The sequences of this oligonucleotide are underlined in Figure 3A. After heat denaturation at $95^{\circ} \mathrm{C}$ for $10 \mathrm{~min}$ the reaction was chilled on ice and 1 unit Taq polymerase and a sense oligonucleotide from the first exon (also underlined) were added and the PCR reaction was continued in $100 \lambda$ total volume. Twenty-five cycles with hybridization at $60^{\circ} \mathrm{C}$ for $2 \mathrm{~min}$, extension at $72^{\circ} \mathrm{C}$ for $2 \mathrm{~min}$ and denaturation at $94^{\circ} \mathrm{C}$ for $2 \mathrm{~min}$ were performed followed by a final $72^{\circ} \mathrm{C}$ extension for $20 \mathrm{~min}$. The PCR products of 450 bp were subcloned and 2 individual isolates were sequenced by the Sanger method using Sequenase ${ }^{\oplus}$ kits.

\section{RESULTS}

\section{Cloning and characterization of the mouse CCK gene}

We used a previously reported cDNA clone from mouse brain to probe a mouse genomic library (13). Among eight separate isolates two types were found. These clones represented by $\lambda$ CCK5 and $\lambda$ CCK7, were found to be overlapping and a restriction map of the mouse CCK gene is shown in Figure 1. All the EcoRI sites within the locus are shown above the line; additional restriction sites which are relevant to specific experiments are below the line (not all of the sites for these enzymes are shown). Southern blots of genomic DNA demonstrated identically sized single bands to those found in the genomic clones (data not shown). Thus the CCK gene is single

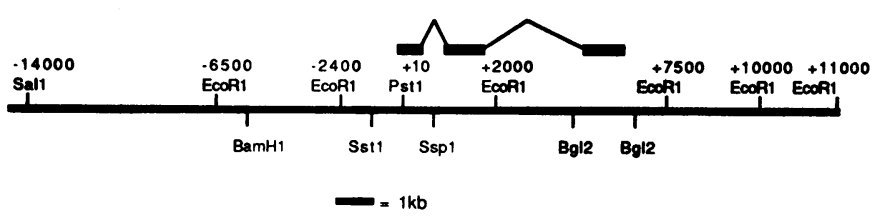

Figure 1. Restriction map of the mouse CCK gene. A mouse genomic library was screened with a mouse brain CDNA and a total of eight genomic clones were isolated. Two different isolates (each represented four times) were found to be overlapping and spanned a region of the mouse CCK gene between -6500 and $+11,000$. An additional genomic subclone extending to $-14 \mathrm{~kb}$ was isolated by rescreening the library. All the EcoRI sites in the locus are shown. The other sites shown (below the line) are noted because they were utilized in subsequent experiments. Only the relevant sites for these restriction enzyme sites are shown. 
copy in the mouse genome. The two original phage clones extended to $-6.5 \mathrm{~kb}$ (the assignment of the RNA start site is described in Figure 2). An additional genomic clone was then isolated which extended to $-14.5 \mathrm{~kb}$ by re-screening the library with a $1200 \mathrm{bp}$ BamHI fragment between -6700 and $-5500 \mathrm{bp}$.

A
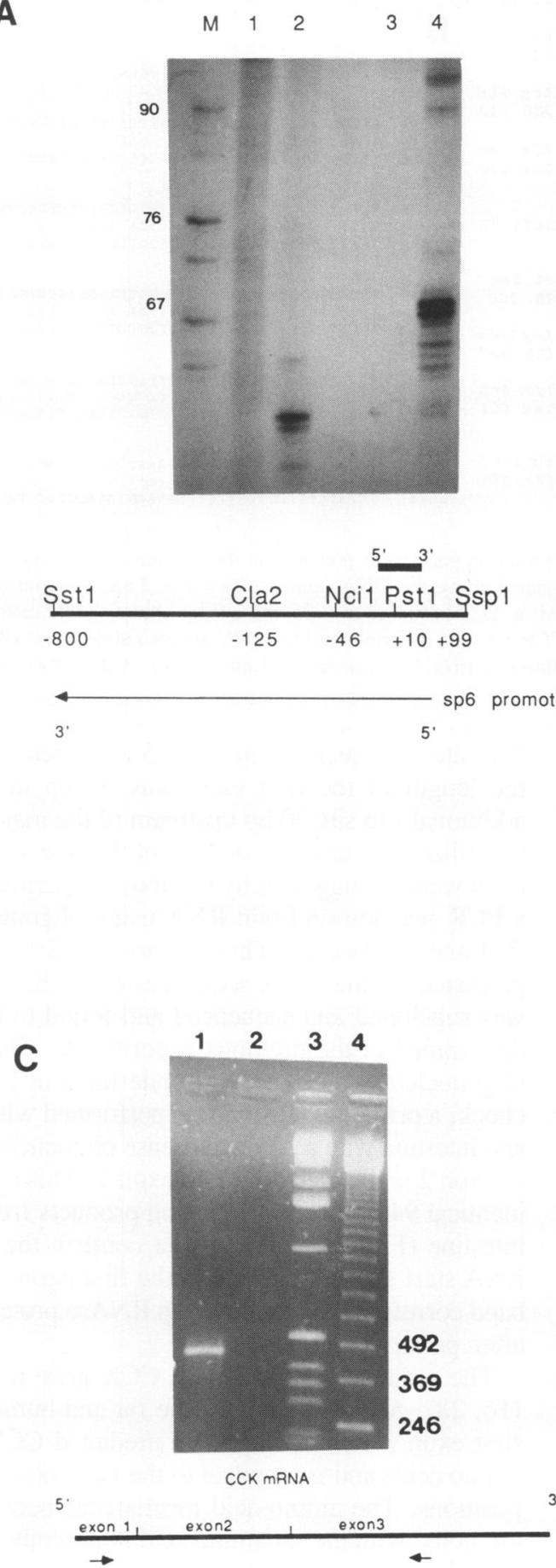

The mouse brain cDNA clone hybridized to two separate restriction fragments from the genomic clones which were separated by $3500 \mathrm{bp}$. The hybridizing regions were sequenced using the dideoxy method and were found to be identical to the mouse cDNA and homologous to the previously cloned rat CCK

B

$\begin{array}{llllll}1 & 2 & 3 & 4 & M\end{array}$

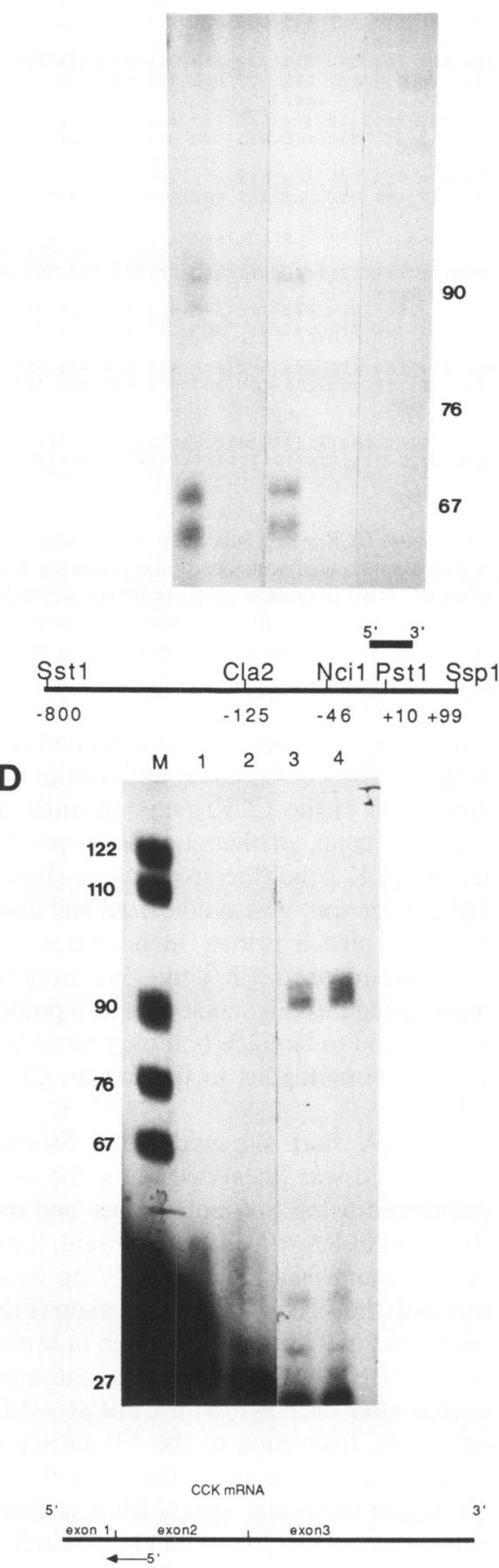

Figure 2. Definition of the RNA start site of the CCK gene. (A) A 900 bp Sst-Ssp fragment was isolated which hybridized to an oligonucleotide specific for the rat CCK first exon. This plasmid was linearized with Sst. An antisense RNA was generated with Sp6 polymerase and hybridized to liver RNA (lane 3) and brain RNA (lane 4) at $59^{\circ}$ trimmed with T2 RNAse and fractionated on an acrylamide gel. In separate experiments the antisense RNA transcript was linearized with PstI and hybridized to the same RNAs from liver (lane 1) and brain (lane 2). (B) The antisense RNA transcripts were linearized with ClaII and hybridized to RNA from brain (lane 1) and liver (lane 2). The same RNAs were then hybridized to an antisense RNA generated after NciI digestion (lane 3, brain; lane 4, liver). (C) Oligonucleotides from exons 1 and 3 were used in PCR reactions together with mouse brain RNA. The sequences of the oligonucleotides are underlined in Figure 3. The PCR reaction yielded the predicted 480 bp DNA fragment, the sequence of which matched the genomic sequence. Lanes 1) brain RNA 2) liver RNA 3) $1 \mathrm{~kb}$ ladder 4) 123 bp ladder. (D) Five micrograms of poly A + RNA were hybridized to a 32 bp oligonucleotide that corresponds to the extreme $5^{\prime}$ end of the second exon and the first $5 \mathrm{bp}$ of the first exon and primer extended using reverse transcriptase. The primer extension products were resolved on a $5 \%$ urea acrylamide gel. The position of the primer relative to CCK RNA is shown at the bottom of the figure. Lanes 1) yeast RNA 2) liver 3) brain 4) intestine. 
A

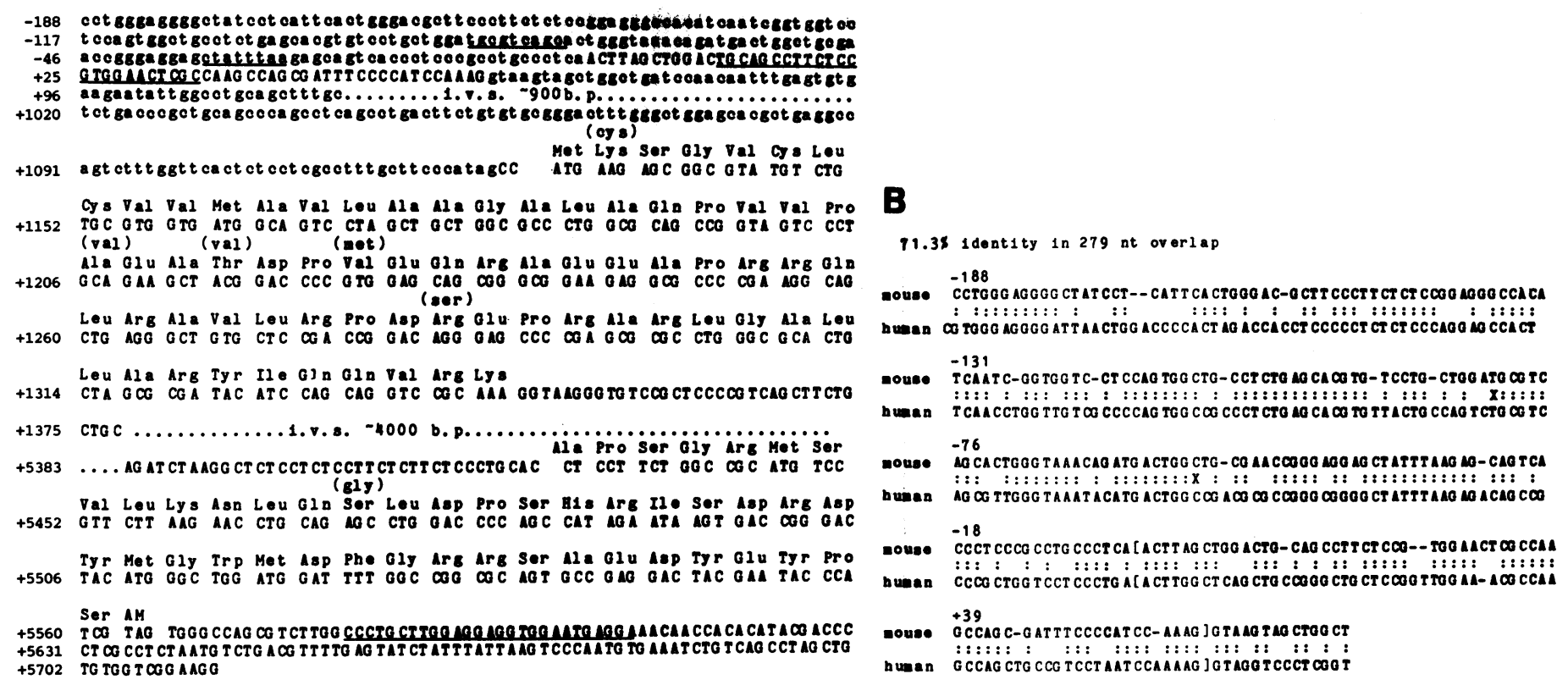

Figure 3. (A) Sequence of the mouse CCK gene. Subclones of the mouse cholecystokinin gene were prepared in the Gemini cloning vector and sequenced using dideoxy sequencing. The predicted amino acid sequence of the precursor is represented above the DNA sequence of exons 2 and 3 . Amino acid mismatches with the rat precursor are in parentheses. Two sequences in the promoter are underlined: a 'tata' sequence at -25 and a 9 bp sequence that matches a cAMP response element of the enkephalin gene. Sequences in the first and third exons used in the PCR reaction described in Figure 2C are also underlined. (B) The DNA sequences at the 5' end of the mouse and human CCK genes were compared using the algorithm described by Pearson and Lipman (19). A dot between the mouse and human sequences indicates identity.

gene $(13,16)$. These sequences corresponded to the second and third exons of the CCK gene. The cDNA clone utilized did not however identify the first exon of the CCK gene. In order to identify the cap site of the CCK gene, an oligonucleotide specific for the first exon of the rat CCK gene (the sequence is shown in the Materials and Methods section) was synthesized and used as a hybridization probe and also a primer in a sequencing reaction with subclones of the mouse CCK gene. We isolated an 800 bp Sst-Ssp fragment, using this oligonuclectide as a probe, and it contained sequences found to be $92 \%$ homologous to the rat CCK promoter and $72 \%$ homologous to the human CCK promoter (Fig. 3; 16, 17).

In order to assign the RNA start site within the Sst-Ssp fragment, the subcloned plasmid was linearized at the Sst site, an RNA probe was synthesized using Sp6 polymerase and the antisense RNA was hybridized to RNA from whole brain. Two closely migrating RNAse resistant fragments of $\sim 67$ bp were found in brain, consistent with the size of the rat first exon (Fig. 2A, lane 4). An additional band of 97 bp was also seen in brain. In order to further localize the RNA start site aditional antisense transcripts were synthesized after cleavage with ClaII at -151 and Nci at -54 . In both cases, truncation of the ${ }^{32} \mathrm{P}$ antisense RNA with these enzymes did not ablate either the 67 or 97 bp bands (Fig. 2B). Thus the higher molecular weight RNA is likely from a start site 30 bp upstream of the lower bands. A similar upstream start has been demonstrated in human (28). These data suggested that a PstI site should be present at +10 bp of the CCK gene. In order to unambiguously position the start ste, an antisense RNA was synthesized from the Sst-Ssp stbclone linearized with PstI. An RNA probe synthesized in this fashion was hybridized to brain RNA and yielded an RNAse resistant band of $57 \mathrm{bp}$ thus assigning the RNA start site to $10 \mathrm{bp}$ upstream of this PstI site (Fig. 2A, lane 2). No other RNAse protected bands could be demonstrated with probes extending $5^{\prime}$ from this
PstI site to sequences up to $14.5 \mathrm{~kb}$. These data suggested that the length of the first exon was $67 \mathrm{bp}$ in brain and that an additional cap site 30 bp upstream of the major cap site can also be utilized. In order to document that the sequences in the first exon were as suggested by the above experiments, we performed a PCR reaction on brain RNA using oligonucleotides from the first and third exons. This reaction yielded a 450 bp product as predicted by the DNA sequences (Fig. 2 C). The PCR product was subcloned and sequenced and found to be identical to that determined in the previous experiments. The sequences of the oligonucleotides utilized are underlined in Figure 3. As a final check, a primer extension was performed with RNA from brain and intestine with a 32 bp antisense oligonucleotide which started in exon 2 and ended $4 \mathrm{bp}$ into exon 1 . This experiment revealed identical 94 bp primer extension products from brain as well as intestine (Fig. 2D). These data confirm the assignment of the RNA start site and the size of the first exon. Curiously only the band corresponding to the 67 bp RNAse protected band was seen after primer extension.

The sequence of the mouse CCK gene is shown in Figure 3 $(16,28)$, As is the case for the rat and human CCK genes, the first exon is non-coding. The predicted CCK precursor is $\mathbf{1 1 5}$ amino acids and is identical to the rat prohormone at all but five positions. The amino acid mismatches between rat and mouse are noted with the rat amino acid in parentheses. Ninety percent homology between the rat and mouse genes was noted in the coding sequence and also in the 190 bp upstream of the DNA start site (data not shown). Seventy-one percent homology was noted in the promoter region between mouse and man and the homology between man and mouse is shown in Figure 3B. The DNA sequences upstream of the RNA start site have many of the features characteristic of eukaryotic promoters. There is a TATTTA box at -25 and a CAAT box at -80 . There is also a perfect $9 \mathrm{bp}$ match between a sequence at -56 of the CCK 

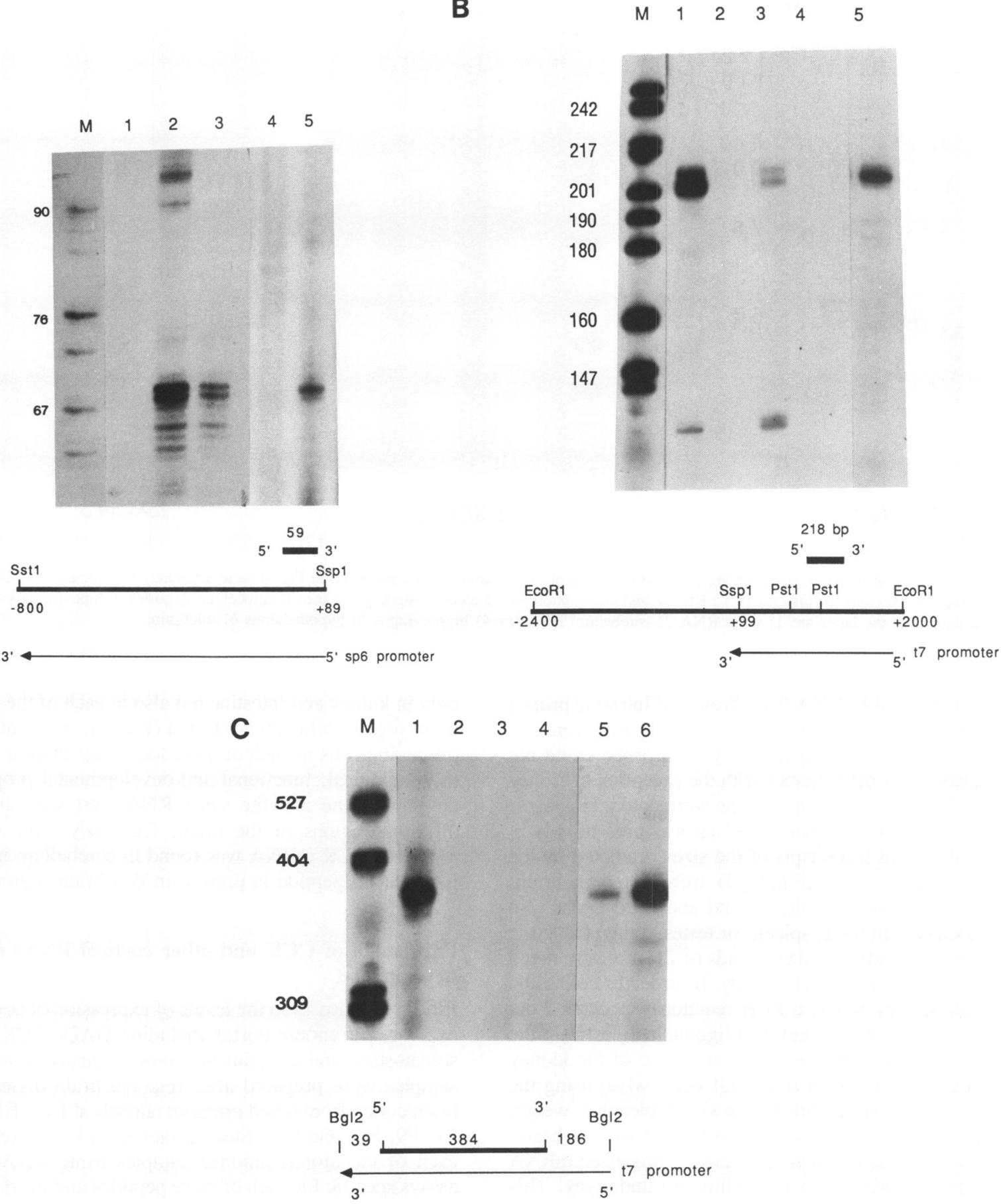

Figure 4. Exon utilization of the CCK gene among tissues of the mouse. Twenty-five micrograms of total RNA were hybridized to each of three different antisense RNAs derived from genomic subclones of the CCK gene corresponding to each of the three exons. Hybridized RNAs were digested with T2 RNAse and fractionated on a 5\% urea acrylamide gel. (A) The same RNA probe described in Fig.2A was hybridized to RNA from 1) liver 2) brain 3) intestine 4) spleen (5) kidney. (B) A 2000 bp RNA probe was generated after digestion of a genomic subclone of the CCK gene with Sspl followed by RNA synthesis with T7 polymerase. The ${ }^{32} \mathrm{P}$ RNA was hybridized to RNA from 1) brain 2) liver 3) kidney 4) spleen 5) intestine. (C) A Bgl2 subclone that included the entire third exon was linearized with SalI. A ${ }^{32}$ P RNA probe was synthesized using T7 polymerase and hybridized to RNA from 1) brain 2) liver 3) spleen 4) testes 5) kidney 6) intestine.

gene and a region of the proenkephalin promoter that has been found to be important in CAMP responsiveness of the proenkephalin gene $(29,30)$.

\section{Exon utilization in brain and other tissues}

In order to define the splice choices utilized for each of the three exons, genomic subclones in pGem1 were utilized that included the Ssp-Sst subclone for exon 1, a $4.5 \mathrm{~kb}$ EcoRI fragment that included the second exon, and a bg12 subclone that spans the third exon (see Fig. 1A). Antisense RNA transcripts from each of these subclones were synthesized using either Sp6 or T7 RNA polymerase and hybridized first to RNA from brain, intestine, kidney and other tissues and subsequently to RNA prepared from cortex, hypothalamus, midbrain, medulla and cerebellum. 

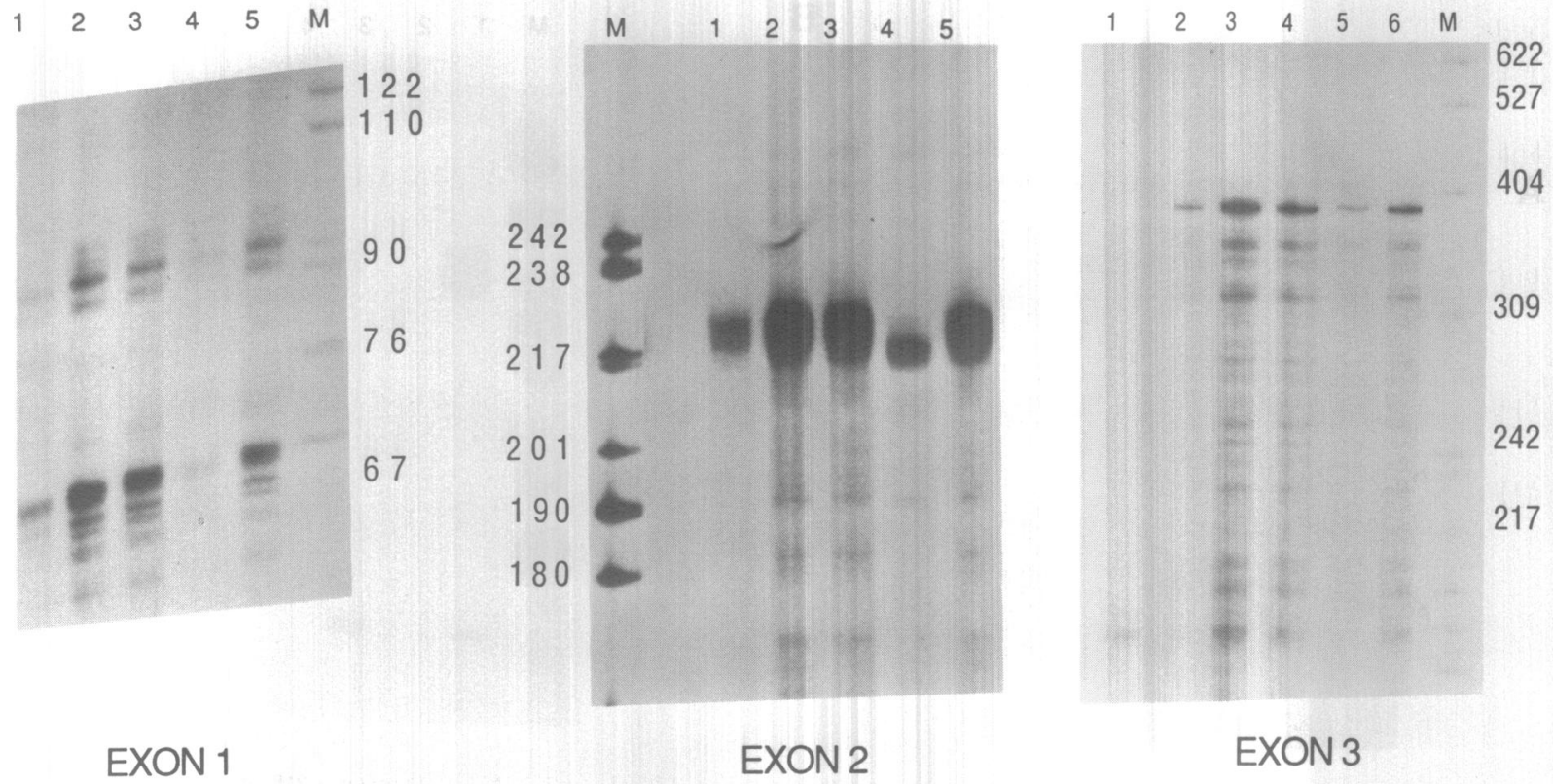

Figure 5. Exon utilization of the CCK gene among different brain regions. The same probes described in Fig. 4 were separately hybridized to RNA prepared from regionally dissected brain regions, trimmed with T2 RNAse and electrophoresed on an acrylamide gel. Lanes 1) cerebellum 2) cortex 3) hippocampus 4) hypothalamus 5) midbrain. In panel (C) the lanes are 1) yeast RNA 2) cerebellum 3) cortex 4) hippocampus 5) hypothalamus 6) midbrain.

As shown in Figure 4A, RNA from brain and intestine protect a 67 bp fragment when an RNA probe specific for exon 1 is utilized. CCK expression using this first exon probe could not be demonstrated in any other tissues with the exception of kidney where levels of $\sim 20 \%$ of that in intestine were noted. However, the higher molecular weight band of $97 \mathrm{bp}$ was present only in brain. Identically sized transcripts of the sizes predicted by the DNA sequences (detailed in Figure 3) were noted in brain, intestine and kidney for both the second and thind exoms. No signals were detected in liver, spleen, or testes. When using the second exon probe identically sized bands of 213 bp were noted in brain, intestine and kidney (Fig. 4B). In order to define the sequence of the kidney RNA, a PCR reaction was carried out using a kidney cDNA library and the oligonucleotides from the first and third exons as described. The sequence of the kidney cDNA was identical to that of brain. Of note, when using the second exon probe, an additional lower molecular weight protected band of $130 \mathrm{bp}$ was seen in kidney as well as brain. This extra band could represent an alternatively processed mRNA and experiments to address this possibility are underway. This band was not present in this particular preparation of intestinal RNA but was present in others (data not shown). When the third exon was utilized identically sized bands of 384 bp were noted among all the expressing tissues, including kidney (Fig. 4C). Even though normal sized RNAs corresponding to each of the three exons were demonstrated in this organ, no CCK immunoreactivity for CCK was demonstrable in a radioimmunoassay of a boiled water extract of mouse kidney (31).

In order to determine the exon utilization and RNA start sites among different brain regions, RNA from regionally dissected cerebellum, cortex, midbrain, hippocampus and hypothalamus was prepared. RNA from each of these brain regions protected the appropriate sizes of RNA (including the putative upstream start site) when using probes specific for each of the three exons. These data demonstrate that the same RNA start site is used not only in kidney and intestine but also in each of the five different brain regions which were tested (Fig. 5). As mentioned, CCKproducing cells in each of these loci differ in their biochemical, morphological, functional and developmental properties. Thus the same gene and the same RNA start sites are utilized in different regions of the brain. Curiously, and as previously reported, CCK mRNA was found in cerebellum in spite of the fact that no peptide is present in this brain region (32).

\section{Expression of CCK and other cortical RNAs during brain development}

Finally we measured the levels of expression of several mRNAs expressed in mouse cortex including GAD, CCK, enkephalin, somatostatin and actin during mouse cortical development. RNA samples were prepared after regional brain dissection of fetal brain cortex from timed pregnant animals at E12, E15, E17, E20, P1, P9, P21 and P28. Steady state RNA levels were assayed in each of the aforementioned samples using RNAse protection assays specific for each of these peptides and the data are shown in Figure 6A.

Low levels of GAD mRNA can be scored at F12 but between F12 and F15 there is a $10 \times$ increase in the mRNA abundance with levels of expression to approximately one-third that seen in adult brain. Post partum there is a thricefold increase with adult levels achieved at 10 days post partum. Among the brain peptides, somatostatin appears first and can be seen to have substantial levels of RNA as early as 15 days of fetal life with an $\sim$ threefold increase to adult level postnatally. CCK first appears at low levels at F18, after somatostatin, followed by a gradual increase over the first 10 days of post natal life. At 10 days post partum, fully adult levels of CCK mRNA were demonstrated. Of the three peptides which were monitored, enkephalin appears last and cannot be demonstrated until 10 days post partum. In these experiments actin remained near constant throughout brain 
A

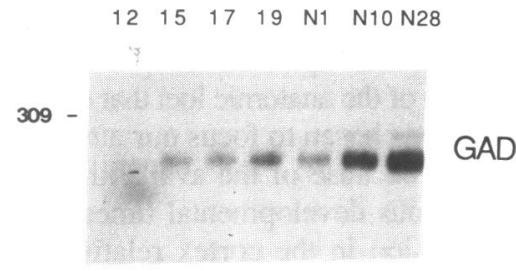

530-
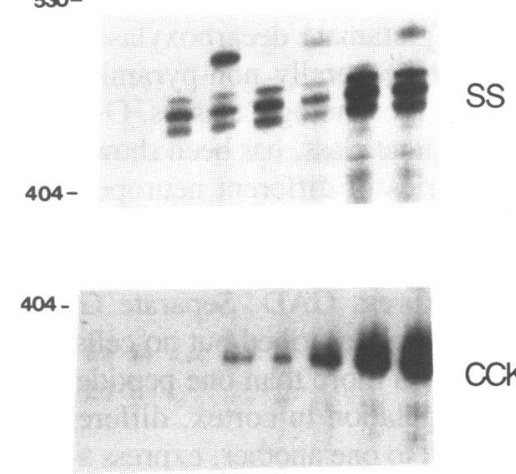

$\begin{array}{lllllllll}\text { B } & 14 & 17 & 19 & 1 & 7 & 10 & 28\end{array}$
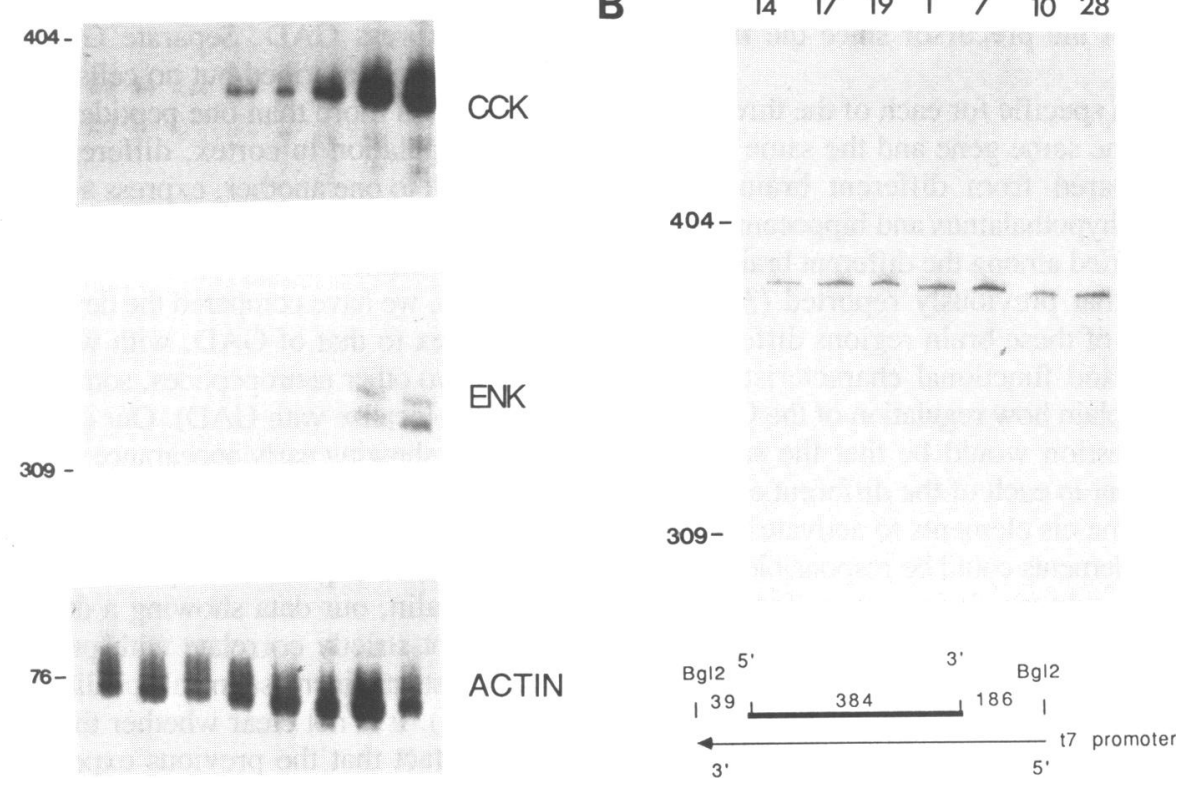

Figure 6. Neuronal RNA expression during cortical development. (A) RNA was prepared from mouse cortex at E12, E15, E17, E19, N1, N10 and N28 using guanidium thiocyanate. Twenty-five $\mu \mathrm{g}$ of RNA were hybridized separately to antisense RNAs specific for glutamate decarboxylase (GAD), somatostatin (SS), cholecystokinin (CCK), enkephalin (ENK) and actin. RNAse resistant hybrids were fractionated on an acrylamide gel. The sizes of the respective protected fragments relative to molecular weight markers are noted. (B) CCK expression during intestinal development. Twenty-five $\mu \mathrm{g}$ RNA were prepared from mouse intestine at F14, F17, F19, N1, N7, N10 and N28 and hybridized to a probe specific for the CCK third exon.

development. Finally we measured the levels of CCK mRNA during intestinal development using the exon 3 probe and found that fully adult levels of the RNA are present before birth in intestine at a time when brain levels are extremely low relative to adult (Fig. 6B).

\section{DISCUSSION}

The mammalian nervous system is composed of an undetermined number of cell types. The diversity of cellular phenotypes demonstrable in the CNS often appears to be the result of a complex pattern of gene expression wherein a particular cell type expresses a unique combination of mRNAs and proteins that are not themselves restricted in their distribution to that cell type (3, 4). This feature of the neuronal phenotype is illustrated by the complex distribution of the neuropeptide cholecystokinin in different brain regions.

Our objective is to elucidate the molecular mechanisms controlling the expression of the CCK gene in each of the cell types that express CCK RNA. A full understanding of the regulation of this gene at each of the various expressing loci would yield insight into the molecular genetic strategies employed when tissue specific genes are expressed in subsets of cell types.

We have previously demonstrated that the CCK gene is present as a single copy in mice and suggested that the same RNA start site is utilized in brain as in small intestine (13). In the present report, we have described the cloning and sequencing of the mouse CCK gene and have made use of genomic subclones to define the exons utilized in each region of brain known to express this RNA. We have found that the RNA from brain protects a doublet of $\sim 67 \mathrm{bp}$ when hybridized to a probe that spans the first exon and concluded that this probe detects the RNA start site. This was confirmed by sequencing of CCK cDNA synthesized from a PCR reaction using oligonucleotides predicted to be in the first and third exons. The evidence that this fragment is the site of transcription initiation of the CCK gene includes 
the extensive homology with the previously cloned rat and human CCK genes in this region, the truncation of the protected band by $10 \mathrm{bp}$ when an antisense RNA probe is restriction digested with PstI (present at +10 on the CCK gene), the presence of characteristic transcription initiation signals such as a 'TATA' box, CAAT box and finally our inability to find RNAse resistant fragments other than those accounted for by the three exons described, including probes that extend from $-14 \mathrm{~kb}$ to +11 $\mathrm{kb}$ on the CCK gene. The length of the first exon was confirmed by primer extension.

We have used cloned probes specific for each of the three CCK exons to demonstrate that the same start site and splice choices are used among brain, intestine and kidney. An additional start site at $\sim-30$ may also be used specifically in brain. It is unclear however why this alternative start site was not demonstrable after primer extension. This upstream start site should not alter the translation reading frame of the precursor since the first exon is untranslated.

We have also used probes specific for each of the three mouse exons to demonstrate that the same gene and the same start site are utilized in RNA prepared from different brain regions including cortex, midbrain, hypothalamus and hippocampus. The abundance of CCK RNA varied among the different brain regions tested and was similar to that previously reported (33). CCK expressing neurons in each of these brain regions differ in their morphologic, biochemical and functional characteristics. Two simple possibilities could explain how regulation of the CCK gene is effected. The first suggestion would be that the same gene regulatory factors are present in each of the different expressing cell types and act on the same cis elements to activate this gene. Alternatively, different cis elements could be responsible for CCK activation in each neuronal subtype. It is known, for example, that multiple regulatory elements are present upstream (or downstream) of eukaryotic genes and that these elements sometimes act independently and allow the same gene to respond independently to many different stimuli. There are precedents for either of the aforementioned possibilities. In Drosophila melanogaster the dopa decarboxylase gene is expressed in the nervous system and the integument (29) and different DNA sequences appear to be involved in regulation of this gene at either site. Also, different DNA sequences are required for the expression of the fushi tarazu gene in nests of neuronal cells and imaginal disks (35).

It is our objective to ascertain whether the same or different DNA sequences confer cell specific expression of the CCK gene in each of the different regions of brain that express this transcript. We have recently characterized several CCK-producing tumor cell lines which should allow the delineation of at least some of the DNA sequences required for cell specific expression of the CCK gene (36). The ability to introduce recombinant DNAs into the germ line of transgenic mice should make it possible to compare the DNA sequences required for expression of the CCK gene in these cultured cells to those which are required in vivo. Moreover, deletion analysis and scoring of a functioning CCK promoter-recorder gene construct in transgenic animals will allow us to conclude whether the same or different cis-acting elements are responsible for CCK expression in each region of brain. It is also possible that posttranslational control is relevant in the control of the CCK gene since CCK mRNA can be detected in cerebellum where no peptide can be detected. A similar disparity between CCK mRNA and CCK peptide has been reported in thalamus (33). It is likewise curious that CCK RNA is detectable in kidney whereas CCK immunoreactivity is absent.
Ultimately we would like to identify the intracellular and extracellular signals that regulate the expression of CCK mRNA in each of the anatomic loci that express this gene. In initial studies we have chosen to focus our attention on CCK expression in the cortex because of the availability of large amounts of material at various developmental times, and the higher levels of CCK expression in the cortex relative to other brain regions.

In cortex $\sim 30-50 \%$ of neurons express GABA (gamma amino butyric acid) and the enzyme responsible for its synthesis, GAD (glutamate decarboxylase) $(9,10)$. Cortical GABAergic cells are generally non-pyramidal cells and consist of several distinct morphologic classes. One class of GABAergic cells, the small round class, has been shown to co-express GABA and each of a series of different neuropeptides. Some cells in this class express GABA and CCK while others express somatostatin and others express neuropeptide $Y(9,10)$. In cortex all CCK positive cells express GAD. Separate GABAergic cells express other peptides as described but no cells in this class have been shown to express more than one peptide. Thus among the GABAergic cell population in cortex, different cells, which are sometimes adjacent to one another, express a different neuropeptide $(9,10)$. We would like to understand the mechanisms that determine peptide choice in the cortical GABAergic cell population.

Thus, we have compared the developmental expression of CCK in cortex to that of GAD, with which it is co-localized, as well as to two other neuropeptides, somatostatin and enkephalin (which also co-localize with GAD). Our data compare well with previous reports showing early appearance of GAD as well as somatostatin (37-42) followed later by the appearance of CCK RNA. CCK immunoreactivity during development also correlates well with CCK RNA expression reported here $(13,43)$. In the case of enkephalin, our data showing a delayed expression of its RNA does not strictly correlate with prior data showing an $\sim 5-10$ fold postnatal increase in enkephalin immunoreactivity in rat brain $(44,45)$. It is not clear whether this difference is a consequence of the fact that the previous experiments were done in rats as opposed to mouse.

The time of appearance of these RNAs are consistent with the possibility that a GABAergic cell population develops early in cortical development and that further differentiation can occur by means of cell specific choice of a particular peptide. This hypothesis must be considered with caution on the basis of this study, however, since the data do not prove that co-expression of GAD and a particular peptide does not occur simultaneously in subsets of cells during development, nor does it prove that the same cell population that expresses these peptides during development expresses these markers during adulthood. These issues can be more directly addressed by the use of in situ hybridization. What is clear, however, is that each of these mRNA is regulated independently during development and that current advances in molecular biology may make it possible to dissect the molecular genetic mechanisms responsible for their control.

\section{ACKNOWLEDGEMENTS}

We would like to thank Dr Bruce S.Schneider for his many helpful discussions. We would also like to thank Lois Cousseau for her expert assistance in preparing this manuscript. J.M.F. is an Assistant Investigator with the Howard Hughes Medical Institute. 


\section{REFERENCES}

1. Derman,E., Krauter,K., Walling,L., Weinberger,C., Ray,M. and Darnell,J.E.,Jr. (1981) Cell 23, 731-739.

2. Powell,D.J., Friedman,J.M., Oulette,A.J., Krauter,K.S. and Darnell,J.E.,Jr. (1984) J. Mol. Biol. 179, 21-35.

3. Clayton,D.F., Huecas,M.E., Sinclair-Thompson,E.Y., Nastiuk,K.L. and Nottebohm,F. (1988) Neuron 1, 249-261.

4. Branks,P.L. and Wilson,M.C. (1986) Mol. Brain Res. 11, 1-16.

5. Miller,F.D., Naus,C., Higgins,G., Bloom,F. and Milner,R. (1987) J. Neurosci. 7, 2433-2444.

6. Innis,R.B., Correa,F.M.A., Uhl,G.R., Schneider,B. and Snyder,S.H. (1979) Proc. Natl. Acad. Sci. USA 76, 521-525.

7. Mutt,V. (1980) In Glass,J.B. (ed.). Gastrointestinal Hormones. Raven, New York, pp. 169-221.

8. Rehfeld,J.F. (1978) J. Biol. Chem. 253, 4022-4030.

9. Hendry,S.H.C., Jones,E.G., DeFelipe,J., Schmechel,D., Brandon,C. and Emson,P.C. (1984) Proc. Natl. Acad. Sci. USA 81, 6526-6530.

10. Somogyi,P., Hodgson,A.J., Smith,A.D., Nunzi,M.G., Gorio,A. and Wu,J.Y. (1984) J. Neurosci. 4, 2590-1603.???

11. Hokfelt,T., Skirboll,L., Rehfeld,J.F., Goldsten,M., Markey,K. and Dann,0. (1980) Neurosci. 5, 2093-2124.

12. Mezey,E., Reisine,T.D., Skirboll,L., Beinfeld,M. and Kiss,J.Z. (1986) Proc. Natl. Acad. Sci. USA 83, 3510-3512.

13. Friedman,J., Schneider,B.S. and Powell,D. (1985) Proc. Natl. Acad. Sci. USA 82, 5593-5597.

14. Costa,R.H., Lai,E. and Darnell,Jr.,J.E. (1986) Mol. Cell. Biol. 6, 4697-4708.

15. Benton,W.D. and Davis,R.W. (1977) Science 196, 180-184.

16. Deschenes,R.J., Haun,R.S., Funckes,C.L. and Dixon,J. (1985) J. Biol. Chem. 260, 1280-1286.

17. Deschenes,R.J., Lorenz,L.J., Haun,R.S., Roos,B.A., Collier,K.J. and Dixon,J.E. (1984) Proc. Natl. Acad. Sci. USA 81, 726-730.

18. Sanger,F., Nicklen,S. and Coulson,A.R. (1977) Proc. Natl. Acad. Sci. USA 74, 5463-5468.

19. Pearson,W.R. and Lipman,D.J. (1988) Proc. Natl. Acad. Sci. USA 85, 2444-2448.

20. Chirgwin,J.M., Pryzbyla,A.E., MacDonald,R. and Rutter,W.J. (1979) Biochemistry 18, 5294-5299.

21. Glowinski,J. and Iversen,L.L. (1966) J. Neurochem. 13, 655-669.

22. Melton,D., Krieg,P.S., Rebagliati,M.R., Maniatis,T., Zinn,K. and Green,M.R. (1984) Nucl. Acids Res. 12, 7035-7056.

23. Kaufman,D.L., McGinnis,J.F., Krieger,N.R. and Tobin,A. (1986) Science 232, $1138-1140$.

24. Montminy,M.R.,Low,M.J., Tapia-Arancibia,L., Reichlin,S., Mandel,G. and Goodman,R.H. (1986) J. Neurosci. 6, 1171-1176.

25. Yoshikawa,K., Williams,C. and Sabol,S.L. (1984) J. Biol. Chem. 259, 14301-14308.

26. Enoch,T., Zinn,K. and Maniatis,T. (1986) Mol. Cell. Biol. 6, 801-810.

27. Friedman,J.M., Babiss,L.E., Clayton,D.F. and Darnell,Jr.,J.E. (1986) Mol. Cell. Biol. 6, 3791-3797.

28. Takahashi,Y., Fukushige,S., Murotsu,T. and Matsubara,K. (1986) Gene 50, $353-360$.

29. Terao,M., Watanabe,Y., Mishina,M. and Numa,S. (1983) EMBO J. 2, 2223-2228.

30. Montminy,M.R., Sevarino,K.A., Wagner,J.A., Mandel,G. and Goodman,R.H. (1986) Proc. Natl. Acad. Sci. USA 83, 6682-6686.

31. Schneider,B., Monahan,J. and Hirsch,J. (1979) J. Clin. Invest. 64, $1348-1356$.

32. Gubler,U., Chua,A.O., Young,D., Fan,Z-W. and Eng,J. (1987) J. Biol. Chem. 262, 15242-15245.

33. Voigt,M.M. and Uhl,G.R. (1988) Mol. Brain Res. 4, 247-253.

34. Scholnick,S.B., Bray,S.J., Morgan,B.A., McCormick,C.A. and Hirsh,J. (1986) Science 234, 998-1002.

35. Hiromi,Y. and Gehring,W.J. (1987) Cell 50, 963-974.

36. Schneider,B.S., Helson,L., Monahan,J.W. and Friedman,J.M. (1989) J. Clin. Endocrin. Metabolism 69, 411-419.

37. Zingg,H.H., Goodman,R.H. and Habener,J.F. (1984) Endocrinology 115 , 90-94.

38. McGregor,G.P., Woodhams,P.L., O'Shaughnessy,D.J., Ghatei,M.A., Polak,J.M. and Bloom,S.R. (1982) Neuroscience Letters 28, 21-27.

39. Shiosaka,S., Takatsuki,K., Sakanaka,M., Inagaki,S., Takagi,H., Senba,E., Kawai,Y., Ilda,H., Minagawa,H., Hara,Y., Matsuzaki,T. and Tohyama,M. (1982) J. Comp. Neurology 204, 211-224.

40. Roberts,E., Harman,P.J. and Frankel,S. (1951) Proc. Soc. Exp. Biol. and Med. 78, 799-803.
41. Bond,R.W., Jansen,K.R. and Gottlieb,D.I. (1988) Proc. Natl. Acad. Sci. USA 85, 3231-3234.

42. Sims,K.L. and Pitts,Jr.,F.N. (1970) J. Neurochem. 17, 1607-1612.

43. Goldman,S.A., Monahan,J.W. and Schneider,B.S. (1985) Dev. Brain Res. 22, 237-246.

44. Schwartz,J.P. and Simantov,R. (1988) Dev. Brain Res. 40, 311-314.

45. Bayon,A., Shoemaker,W.J., Bloom,F.E., Mauss,A. and Guillemin,R. (1979) Brain Res. 179, 93-101. 